\title{
Lumbosacral pedicle screw placement using a fluoroscopic pedicle axis view and a cannulated tapping device
}

Toshitaka Yoshii ${ }^{1,2 *}$, Takashi Hirai ${ }^{1,2}$, Tsuyoshi Yamada ${ }^{1,2}$, Satoshi Sumiya', Renpei Mastumoto ${ }^{1,2}$, Tsuyoshi Kato $^{1}$, Mitsuhiro Enomoto ${ }^{1,2}$, Hiroyuki Inose ${ }^{1,2}$, Shigenori Kawabata ${ }^{1}$, Kenichi Shinomiya ${ }^{1,2}$ and Atsushi Okawa $a^{1,2}$

\begin{abstract}
Background: Pedicle screw insertions are commonly used for posterior fixation to treat various spine disorders. However, the misplacement of pedicle screws can lead to disastrous complications. Inaccurate pedicle screw placement is relatively common even when placement is performed under fluoroscopic control. In order to improve the accuracy of the screw placement, we applied a technique using guide wires and a cannulated tapping device with the assistance of a fluoroscopic pedicle axis view.

Methods: From 2006 to 2011, 854 pedicle screws were placed in 176 patients in lumbosacral spinal fusion surgeries. The accuracy of screw placement was evaluated using postoperative reconstructed computed tomography images. Screw misplacement was classified as minor (cortical perforation $<3 \mathrm{~mm}$ ), moderate (cortical perforation 3-6 mm), or severe (cortical perforation $>6 \mathrm{~mm}$ ). Using logistic regression analysis, we also investigated the potential risk factors associated with screw misplacement.

Results: Pedicle screw misplacement was observed in 37 screws (4.3\%) in 34 patients. In the sub-classification analysis, 28 screws (3.3 \%) were determined to be minor perforations, 7 screws (0.8 \%) were considered to be moderate perforations, and 2 screws $(0.2 \%)$ was judged to be a severe perforation (cortical perforation $>6$ mm). None of the 28 screws that were considered to be minor perforations were associated with any significant symptoms in the patients. However, 2 of the 9 screws that were determined to be moderate or severe perforations caused neurological symptoms (1 of which required revision). No significant differences were observed in the incidence of screw misplacement among the vertebral levels. Significant risk factors for screw misplacement were obesity and degenerative scoliosis. The odds ratios of these significant risk factors were 3.593 (95\% confidence interval (Cl), 1.061-12.175) for obesity and 8.893 for degenerative scoliosis (95 \% Cl, 1.200-76.220).
\end{abstract}

Conclusions: A modified fluoroscopic technique using a pedicle axis view and a cannulated tapping instrument can achieve safe and accurate pedicle screw placement. In addition, obesity and degenerative scoliosis were identified as significant risk factors for screw misplacement.

Keywords: Pedicle screw placement, Accuracy, Pedicle axis view, Cannulated tapping, Risk factor

\footnotetext{
* Correspondence: yoshii.orth@tmd.ac.jp

'Department of Orthopaedic Surgery, Graduate School, Tokyo Medical and Dental University, 1-5-45 Yushima, Bunkyo-ku, Tokyo 113-8519, Japan ${ }^{2}$ Section of Regenerative Therapeutics for Spine and Spinal Cord, Graduate School, Tokyo Medical and Dental University, 1-5-45 Yushima, Bunkyo-ku, Tokyo 113-8519, Japan
} 


\section{Background}

Pedicle screw insertions are commonly used for posterior fixation to treat various spine disorders, deformities, and trauma. However, the misplacement of pedicle screws can lead to disastrous complications because of the close proximity to neural tissue and the surrounding vessels, although rare, serious complications have been reported, such as dural tear, nerve-root irritation, neural injury, epidural hematoma causing neurological deficit, and vascular violation including aortic abutment [1]. Prior work has shown that the accuracy of pedicle screw insertion is crucial for the efficiency and stability of this surgical procedure [2]. Therefore, the accurate and safe placement of screws within the pedicle is critically important during surgeries.

To ensure the accurate insertion of pedicle screws, various conventional techniques focusing on anatomical landmarks, entry points, and insertion angles have been introduced. Intraoperative fluoroscopic guidance has also been used to avoid screw misplacement. However, inaccurate pedicle screw placement is relatively common even when placement is performed under fluoroscopic control, and it can result in vascular or neurological problems [3-6]. To improve the accuracy of the screw placement, we modified the conventional intraoperative fluoroscopic guidance procedure. Specifically, we applied a technique using guide wires and a cannulated tapping device with the assistance of a fluoroscopic pedicle axis view to confirm the positioning of the guide wires. Here, we introduce this pedicle screw insertion technique and show the accuracy of screw placement for lumbosacral fixation, as well as potential risk factors affecting screw misplacement.

\section{Materials and methods}

This is a retrospective case series study approved by an institutional review board (Tokyo Medical and Dental University Research Ethical Committee. No. 1775). We examined 176 patients (69 males and 107 females, mean age $65.5 \pm 14.0$ years at the time of surgery) who underwent lumbosacral spinal fusion between 2006 and 2011. The indications for surgery were isthmic spondylolisthesis in 14 cases, degenerative spondylolisthesis in 63 cases, lumbar spinal canal stenosis with degenerative instability in 77 cases, degenerative scoliosis in 13 cases, and vertebral fracture in 9 cases (Table 1). The patients were evaluated preoperatively using magnetic resonance imaging and computed tomography $(\mathrm{CT})$; adequate decompressive procedures, including laminectomy, facetectomy, and discectomy, were performed if necessary. A total of 854 pedicle screws were inserted into the lumbosacral vertebrae: 30 screws in L1, 48 screws in L2, 104 screws in L3, 284 screws in L4, 320 screws in L5, and 68 screws in S1 (Table 1). Legacy pedicle screw systems (Medtronic, Minneapolis, MN, USA) were used for posterior spinal stabilization.
Table 1 Patients' data

\begin{tabular}{ll}
\hline$N$ & 176 \\
\hline Age (years old) & $65.5 \pm 14.0$ (range: 20-83) \\
Male/female & $69 / 107$ \\
Disease & \\
$\quad$ Isthmic spondylolisthesis & 14 \\
Degenerative spondylolisthesis & 63 \\
Spinal canal stenosis & 77 \\
Degenerative scoliosis & 13 \\
Vertebral fracture & 9 \\
Level of pedicle screw placement & \\
L1 & 30 \\
L2 & 48 \\
L3 & 104 \\
L4 & 284 \\
L5 & 320 \\
S1 & 68 \\
Total & 854 \\
\hline
\end{tabular}

\section{Procedure}

The patient was placed in a prone position on an operating table with a radiolucent four-point frame. Following standard surgical exposure with a midline incision and bilateral exposure of the transverse processes, the position of the pedicle was estimated using anatomical landmarks $[7,8]$ and the preoperative CT images. The dorsal aspect of the pedicle was decorticated using an air drill and was typically cannulated using a thin gearshift probe or a curette to a depth of approximately $30 \mathrm{~mm}$. After the guide wire (diameter: $1.5 \mathrm{~mm}$ ) was placed in the pilot hole, a multiplanar fluoroscope was used to obtain pedicle axis views (Fig. 1a, b). To obtain a fine pedicle axis view, the $\mathrm{C}$-arm was correctly tilted in the cranial/ caudal direction (sagittal plane) to ensure that the superior endplate of the vertebral body was parallel to the image intensifier's beam, and it was rotated in the anterior-posterior (AP)/lateral direction (axial plane) to ensure that the pedicle cortex wall could be clearly visualized (Fig. 2a). The correct positioning of the guide wire was confirmed using this pedicle axis view [9]. Further confirmation of the guide wire position was made using a lateral view (Fig. 2b). After modifying the trajectory (if necessary), the pedicle was tapped with a cannulated tapping device passing over the guide wire. The guide wire could be visualized in the cannulated tap to ensure that the wire does not move forward and penetrate the anterior wall of the vertebral body (Fig. 1c). Following this process, an adequately sized pedicle screw was inserted into place. No intraoperative neurophysiological monitoring was performed for the screw insertion. 

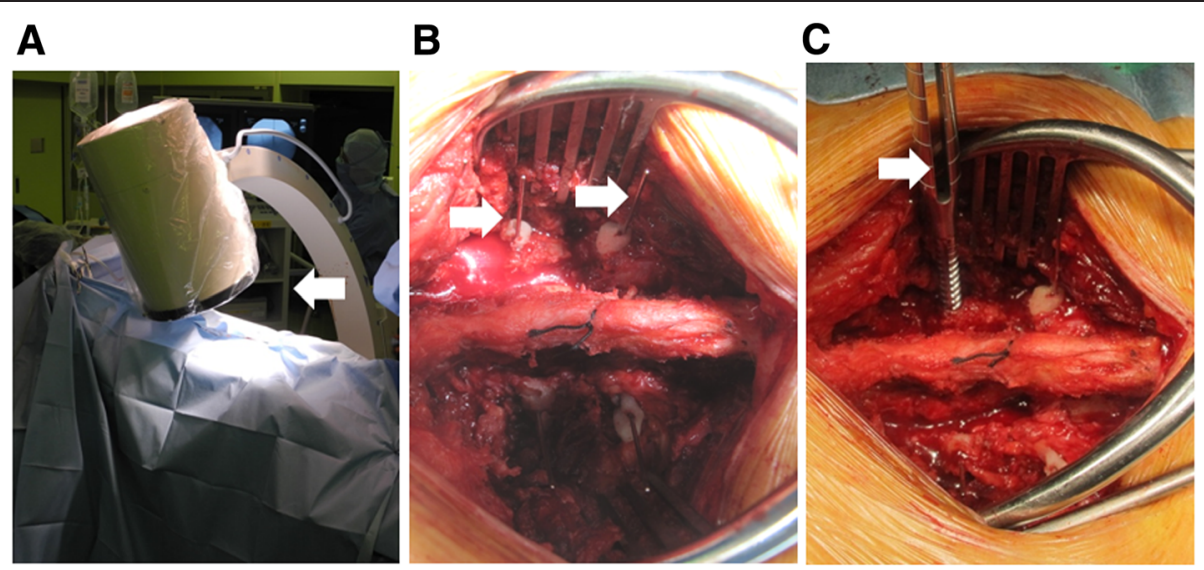

Fig. 1 a Fluoroscopic pedicle axis view. The C-arm was rotated in the anterior-posterior (AP)/lateral direction (arrow) to ensure that the pedicle cortex wall could be clearly visualized. b Guide wires $(1.5 \mathrm{~mm}$ ) were placed in the pilot holes (arrows). c A cannulated tapping device. The arrow: the guide wire can be visualized in the cannulated tap to ensure that the wire does not penetrate the anterior wall

\section{Evaluations of the screw position}

Within 2 weeks of surgery, postoperative CT scans were obtained for all patients to assess the implant position using a 64-row multidetector CT unit (TOSHIBA Medical, Tokyo, Japan), which can provide high-resolution images (in-plane resolution of $0.31 \mathrm{~mm}$ ). The series consisted of 0.5 -mm-thick CT sections that were acquired in helical mode and were reconstructed at $0.5-\mathrm{mm}$ intervals. The acquisition parameters were $120 \mathrm{kV}$ and $400 \mathrm{~mA}$. The raw data were reconstructed with axial 2.0-mm-thick CT sections every $2 \mathrm{~mm}$ with a field of view that was adequate for spine visualization and for sagittal and coronal reconstructions of the lumbar spine.

The screw position was assessed by two independent observers. Screw placement was considered to be correct when the screw was completely surrounded by the pedicle, and no portion of the screw perforated outside the cortex. Screw misplacement was classified according to the classification system suggested by Schizas et al. [10], and misplacements were defined as minor (cortical perforation $<3 \mathrm{~mm}$ ), moderate (cortical perforation 3-6 $\mathrm{mm}$ ), or severe (cortical perforation $>6 \mathrm{~mm}$ ) (Fig. 3a-d). We generally penetrate the anterior cortex in S1 screws in osteoporotic cases: such bicortical screws were not considered to be screw misplacements. The direction of misplacement was divided into the following four types: medial, lateral, inferior, and superior. Potential factors affecting screw misplacement (i.e., age, gender, obesity, levels of fusion, and diseases) were also evaluated using logistic regression analysis; obesity was defined as having a body mass index (BMI) $\geq 25$ or $<25$.
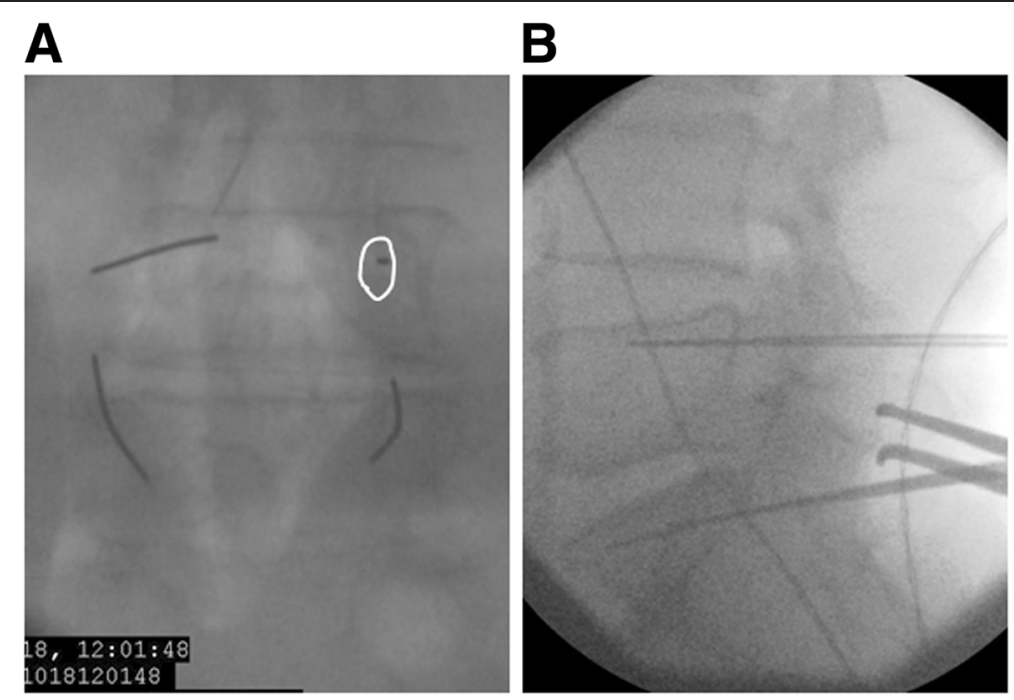

Fig. 2 a Fluoroscopic pedicle axis view; (b) fluoroscopic lateral view 
A

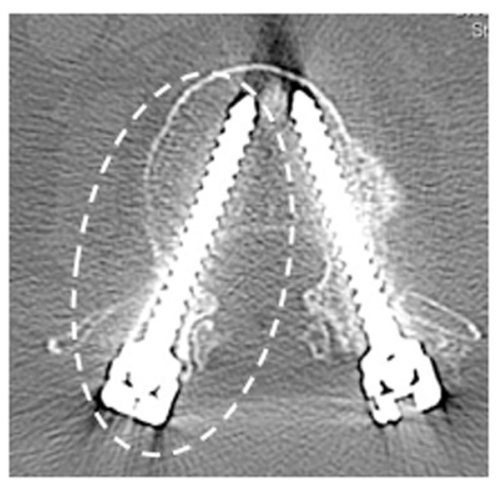

C

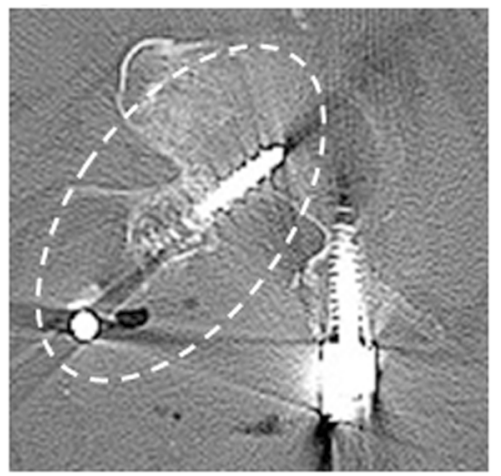

B

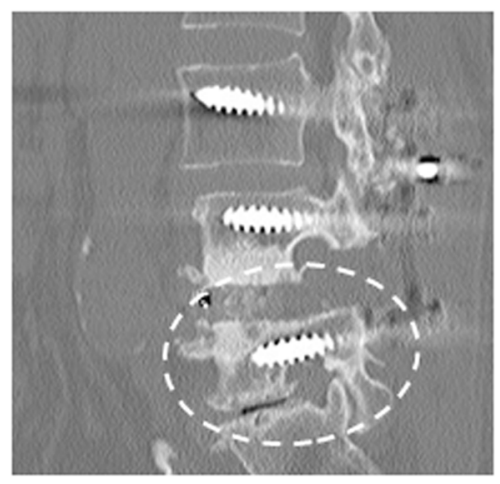

D

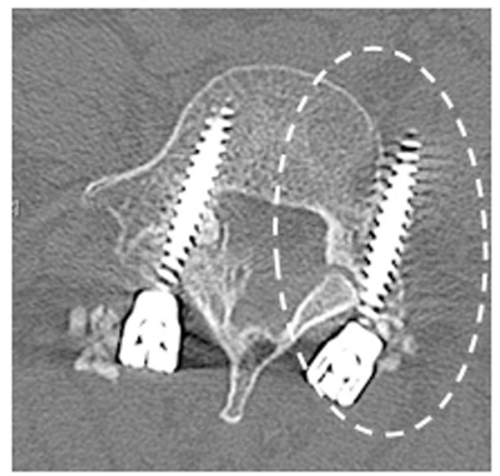

Fig. 3 The pedicle screws judged as misplacement. a Medial minor perforation, (b) inferior minor perforation, (c) medial moderate perforation, and (d) lateral severe perforation

\section{Statistical analysis}

Chi-square tests were used to compare the incidence of screw misplacement. The association between the dichotomous outcome variables (correct or incorrect) and the independent variables were analyzed using logistic regression methods, and the adjusted odds ratios (ORs) with $95 \%$ confidence intervals (CIs) were calculated. Inter-observer agreement was measured using kappa coefficient scores. The significance threshold was set at $p<0.05$. Statistical analyses were performed using the commercial software package SPSS ver. 19 (SPSS, Chicago, IL, USA).

\section{Results}

In our assessment of 854 pedicle screws inserted in 176 patients, 817 screws $(95.7 \%)$ were determined to be adequately inserted. Misplacement of the pedicle screw was observed in 37 screws (4.3\%) in 34 patients. In the subclassification analysis, 28 screws $(3.3 \%)$ were considered to be minor perforations (cortical perforations $<3 \mathrm{~mm}$ ), 7 screws $(0.8 \%)$ were observed to exhibit moderate perforations (cortical perforations $3-6 \mathrm{~mm}$ ), and 2 screws $(0.2 \%)$ was judged to have a severe perforation (cortical perforation $>6 \mathrm{~mm}$ ) (Table 2). There was substantial inter-observer agreement in judging screw positions, and the observed kappa score was 0.71. Regarding the distribution of screw misplacements at the vertebral level, 2 of 30 (6.7 \%) screws were observed in L1, 5 of 48 (10.4 \%) were observed in L2, 4 of 104 (3.8 \%) were observed in L3, 12 of 284 (4.2\%) were observed in L4, 12 of 320 (3.8\%) were observed in L5, and 2 of 68 (2.9\%) were observed in S1 (Table 3). The incidence of screw misplacement tended to be greater in the upper lumbar spine, but no statistically significant differences in screw misplacement were observed $(p=0.38)$. The screw misplacement direction was lateral for 17 screws $(45.9 \%$ of

Table 2 Interpretation of pedicle screw placement

\begin{tabular}{ll}
\hline Screw placement & No. of screws (\%) \\
\hline Adequate insertion & $817(95.7)$ \\
Misplacement & $37(4.3)$ \\
$\quad$ Minor (cortical perforation $<3 \mathrm{~mm})$ & $28(3.3)$ \\
Moderate (cortical perforation $3-6 \mathrm{~mm})$ & $7(0.8)$ \\
$\quad$ Severe (cortical perforation $>6 \mathrm{~mm})$ & $2(0.2)$ \\
\hline
\end{tabular}

No. number 
Table 3 Distribution of screw misplacement

\begin{tabular}{ll}
\hline & No. of misplaced screws (\%) \\
\hline Level & $2(6.7)$ \\
L2 & $5(10.4)$ \\
L3 & $4(3.8)$ \\
L4 & $12(4.2)$ \\
L5 & $12(3.8)$ \\
S1 & $2(2.9)$ \\
Direction & 1 \\
Superior & 1 \\
Inferior & 3 \\
Medial & 16 \\
Lateral & 17 \\
\hline No. number &
\end{tabular}

the misplaced screws), medial for 16 screws (43.2\%), superior for 1 screw (0.3\%), and inferior for 3 screws $(0.8 \%)$ (Table 3). Of the 28 screws that were judged to be minor perforations, none were associated with any symptoms in the patients. However, for the 9 screws considered to be moderate or severe perforations, 2 screws, 1 of which required revision, caused neurological symptoms.

Logistic regression analysis of screw misplacements was performed to identify the risk factors affecting the accuracy of screw placement. The OR and $95 \% \mathrm{CI}$ for each variable are shown in Table 4. The two factors that were significantly associated with the risk of screw misplacement were obesity and degenerative scoliosis. The ORs for these significant risk factors were 3.593 (95\% CI, 1.061-12.175) for obesity and 8.893 (95 \% CI, 1.20076.220) for degenerative scoliosis.

\section{Discussion}

Pedicle screws can provide secure fixation of the vertebra and are frequently used to treat various spine disorders and deformities. However, the insertion of pedicle screws remains technically demanding because of the complicated three-dimensional anatomy and the proximity of neurologic and vascular structures. Medially misplaced pedicle screws increase the risk of neurological complications, while lateral misplacements can increase the risk of vascular complications and biomechanical weakness [1, 4]. Therefore, the accurate placement of pedicle screws is critically important to avoid these complications. To minimize the incidence of screw misplacements, a variety of pedicle screw placement procedures has been reported, including the free-hand technique and the use of intraoperative imaging tools, such as plain radiography, fluoroscopy, or image-guided navigation systems [3, 4, 11-15].

Recently, a number of authors have focused on complex three-dimensional navigation techniques to achieve safe screw insertion. Although these studies demonstrate that navigation systems can reduce the incidence of screw misplacement, these types of systems increase the cost and setup time requirements. Several authors have reported that fluoroscopic guidance can improve the accuracy and safety of pedicle screw placement $[4,13,16$, 17]. Lateral C-arm fluoroscopy imaging is useful because it offers real-time two-dimensional views. However, lateral imaging only provides information in the sagittal plane, not in the axial plane. Because screw misplacement most commonly occurs in the axial plane (i.e., medial or lateral) [18], lateral imaging is not sufficient to ensure complete safety when inserting an implant into a complex three-dimensional structure.

In this study, we applied simple fluoroscopic coaxial imaging techniques using a pedicle axis view to confirm the positioning of the guide wires. Because morphologic

Table 4 Logistic regression analysis for screw misplacement

\begin{tabular}{llll}
\hline & Odds ratio & $95 \%$ confidence interval & $p$ value \\
\hline Age & 1.006 & $(0.967-1.047)$ & 0.77 \\
Male/female & 0.942 & $(0.425-2.516)$ & 0.94 \\
Obesity (BMI $\geq$ or $<25)^{*}$ & 3.593 & $(1.061-12.175)$ & 0.040 \\
Single-level/multi-level & 3.683 & $(0.664-20.418)$ & 0.14 \\
No. of vertebrae & 0.43 & $(0.150-1.254)$ & 0.12 \\
Disease & & & 0.23 \\
Isthmic spondylolisthesis & 1.000 & & 0.088 \\
$\quad$ Degenerative spondylolisthesis & 0.419 & $(0.101-1.729)$ & 0.046 \\
Spinal canal stenosis & 0.292 & $(0.071-1.200)$ & 0.89 \\
Degenerative scoliosis* & 8.893 & $(1.200-76.220)$ & $(0.123-6.239)$ \\
Vertebral fracture & 0.875 & &
\end{tabular}


studies have shown that the pedicle axis angle is high in the horizontal plane at the lower lumbar spine (up to $\left.44.0^{\circ}\right)[8,19,20]$, imaging inclined based on the pedicle axial angle is useful to confirm the trajectory of the guide wire. As Yukawa et al. have previously reported, this pedicle axis view is efficacious in determining the appropriate entry point and the trajectory angle for each vertebra, even for the placement of cervical pedicle screws [9]. We also used a cannulated tapping device that is commonly applied in percutaneous pedicle screw systems and is known to be useful to ensure accurate screw placements [21]. Once the guide wire is correctly placed, the process of tapping and screw insertion becomes easier and safer. We modified the conventional fluoroscopic guidance technique by using a pedicle axis view and a cannulated tapping device to achieve accurate pedicle screw insertion.

Thus, a misplacement rate of only $4.3 \%$ was observed in the postoperative two-dimensional reconstructed CT images obtained in this study. Moderate or severe perforations (cortical perforation $\geq 3 \mathrm{~mm}$ ) were observed in only $1.1 \%$ of the inserted pedicle screws, and symptomatic screw malposition occurred in only $0.2 \%$. Previously, Castro et al. investigated a total of 123 pedicle screws placed in 30 patients using fluoroscopy with anteroposterior and lateral projections [3]. Postoperative CT imaging revealed that $40 \%$ of the screws had penetrated the cortex of the pedicle. In a systematic review of studies investigating pedicle screw insertion, the accuracy of screw placement using conventional fluoroscopic assistance was reported to be $28-85 \%$ [4]. The accuracy was improved to $95.7 \%$ with our modified fluoroscopic method, which used a pedicle axis view and a cannulated tapping device. The percentage of accurate screw placements using our technique was comparable to that using recently reported techniques involving fluoroscopybased (81-92\%) or CT-based (89-100\%) navigation [4]. Based on these results, it appears that the modified fluoroscopic technique used in this study can enable accurate and safe screw insertion without requiring special devices, such as navigation systems.

Previous studies have reported that obesity adversely affects the rate of intraoperative complications associated with lumbar fusion surgery [18, 22]. Consistent with these reports, we noted that screw misplacement was significantly affected by the BMI of the patients during our analysis of the risk factors associated with screw misplacements. This result suggests that technical errors can occur because surgical procedures in obese individuals must be performed in a deeper plane with limited visualization. Degenerative scoliosis was another factor that was observed to increase the risk of screw misplacement. In this study, the rate of screw misplacement in the 13 patients with degenerative scoliosis was as high as
$10.4 \%$. It is known that screw placements in deformed spines can be difficult $[23,24]$ because of the rotation of the vertebrae and/or bone sclerosis at the pedicles on the concave side. Pedicle axis views are thought to be useful in identifying the pedicle direction in rotated vertebrae. However, misdirection can still occur in highly rotated vertebrae (Fig. 3c). Furthermore, bone sclerosis can disturb the insertion of guide wires to the appropriate depth and can cause misdirected tapping even when a cannulated tap is used (Fig. 3b). Extensive caution should be used when placing lumbosacral pedicle screws in obese patients and in individuals with degenerative scoliosis. We found that lateral perforation was the most common in the technique used in this study (Table 3, Fig. 3d). Since lateral perforation can cause serious complications such as iatrogenic neural and vascular injury, reconfirmation after screw insertion would be needed using fluoroscopic $\mathrm{A}-\mathrm{P}$ and pedicle axis views especially for the rotated vertebrae in degenerative scoliosis.

The accuracy of pedicle screw placement has been the subject of a number of previous studies [3, 4, 6, 17, 25]. In these studies, the rates of screw misplacement were highly variable, in part, because of the different evaluation methods employed. Most studies have shown that $\mathrm{CT}$ scanning is more accurate than conventional radiography in evaluating screw misplacement. The discrepancy between radiographs and CT images is most striking in medially misplaced screws, for which CT imaging can detect many more cortical penetrations compared to conventional radiographs $[6,26]$. In this study, for all patients, we obtained thin-sliced-high resolution reconstructed CT images using a 64-row multidetector CT system. Screw placement evaluations were made using the simple classification system suggested by Schizas [10]. Therefore, the inter-observer reliability regarding determinations of screw misplacement exhibited good agreement (i.e., a kappa score of 0.71 , which is similar to that reported by Schizas et al. [10]). The precise evaluation method, which utilized a modified fluoroscopic procedure, was employed; it achieved a high level of accuracy for the placement of lumbosacral pedicle screws.

This study has several limitations, such as its retrospective, single-center design and use of radiation exposure. In addition, the investigation was limited to the lumbosacral spine. This study does not include other radiological outcomes, such as the rates of fusion or screw failure. Despite these limitations, the technique used in this study was shown to achieve safe pedicle screw placement with a level of accuracy that is comparable to that observed using techniques that employ navigation systems.

\section{Conclusions}

A modified fluoroscopic technique using a pedicle axis view and a cannulated tapping device can achieve the 
safe and accurate placement of pedicle screws. The accuracy of this technique was shown to be higher than that of conventional fluoroscopy-guided insertions and was comparable to the accuracy achieved using techniques featuring navigation systems. Obesity and degenerative scoliosis were identified as significant factors leading to an increased risk of screw misplacement.

\section{Abbreviations}

Cl: confidence intervals; CT: computed tomography; OR: odds ratio.

\section{Competing interests}

The authors declare that they have no competing interests.

\section{Authors' contributions}

TYO and AO conceived of the study participated in its design and coordination. TYa, SS, RP, and HI collected the data. ME, TK, SK, and KS participated in devising the technique of screw insertion and the tapping device. HT worked on the revision of the manuscript. All authors read and approved the final manuscript.

\section{Authors' information}

TYa is a junior associate professor in Tokyo Medical and Dental University and a member of Japanese Spinal Instrumentation Society. KS is the former president of Japanese Orthopaedic Association.

$\mathrm{AO}$ is a member of board of directors in Japanese Orthopaedic Association.

\section{Acknowledgments}

This work was supported by Health Labour Sciences Research Grant.

Received: 27 January 2015 Accepted: 14 May 2015

Published online: 28 May 2015

\section{References}

1. Hicks JM, Singla A, Shen FH, Arlet V. Complications of pedicle screw fixation in scoliosis surgery: a systematic review. Spine (Phila Pa 1976). 2010;35:E465-70.

2. Grauer JN, Vaccaro AR, Brusovanik G, Girardi FP, Silveri CP, Cammisa FP, et al Evaluation of a novel pedicle probe for the placement of thoracic and lumbosacral pedicle screws. J Spinal Disord Tech. 2004;17:492-7.

3. Castro WH, Halm H, Jerosch J, Malms J, Steinbeck J, Blasius S. Accuracy of pedicle screw placement in lumbar vertebrae. Spine (Phila Pa 1976). 1996;21:1320-4

4. Gelalis ID, Paschos NK, Pakos EE, Politis AN, Arnaoutoglou CM, Karageorgos AC, et al. Accuracy of pedicle screw placement: a systematic review of prospective in vivo studies comparing free hand, fluoroscopy guidance and navigation techniques. Eur Spine J. 2012;21:247-55.

5. Gertzbein SD, Robbins SE. Accuracy of pedicular screw placement in vivo. Spine (Phila Pa 1976). 1990;15:11-4.

6. Laine T, Makitalo K, Schlenzka D, Tallroth K, Poussa M, Alho A. Accuracy of pedicle screw insertion: a prospective $C T$ study in 30 low back patients. Eur Spine J. 1997:6:402-5.

7. Roy-Camille R, Saillant G, Mazel C. Internal fixation of the lumbar spine with pedicle screw plating. Clin Orthop Relat Res. 1986:7-17

8. Weinstein JN, Rydevik BL, Rauschning W. Anatomic and technical considerations of pedicle screw fixation. Clin Orthop Relat Res. 1992;34-46

9. Yukawa $Y$, Kato F, Ito K, Horie Y, Hida T, Nakashima H, et al. Placement and complications of cervical pedicle screws in 144 cervical trauma patients using pedicle axis view techniques by fluoroscope. Eur Spine J. 2009;18:1293-9.

10. Schizas C, Michel J, Kosmopoulos V, Theumann N. Computer tomography assessment of pedicle screw insertion in percutaneous posterior transpedicular stabilization. Eur Spine J. 2007;16:613-7.

11. Kosmopoulos V, Schizas C. Pedicle screw placement accuracy: a meta-analysis. Spine (Phila Pa 1976). 2007:32:E111-20.

12. Karapinar L, Erel N, Ozturk H, Altay T, Kaya A. Pedicle screw placement with a free hand technique in thoracolumbar spine: is it safe? J Spinal Disord Tech. 2008:21:63-7.
13. Idler C, Rolfe KW, Gorek JE. Accuracy of percutaneous lumbar pedicle screw placement using the oblique or "owl's-eye" view and novel guidance technology. J Neurosurg Spine. 2010;13:509-15.

14. Dede O, Ward WT, Bosch PP, Bowles AJ, Roach JW. Using the freehand pedicle screw placement technique in adolescent idiopathic scoliosis surgery: what is the incidence of neurological symptoms secondary to misplaced screws? Spine (Phila Pa 1976). 2014;39:286-90.

15. Park JH, Jeon SR, Roh SW, Kim JH, Rhim SC. The safety and accuracy of freehand pedicle screw placement in the subaxial cervical spine: a series of 45 consecutive patients. Spine (Phila Pa 1976). 2014;39:280-5.

16. Dickman CA, Fessler RG, MacMillan M, Haid RW. Transpedicular screw-rod fixation of the lumbar spine: operative technique and outcome in 104 cases. J Neurosurg. 1992;77:860-70.

17. Amato V, Giannachi L, Irace C, Corona C. Accuracy of pedicle screw placement in the lumbosacral spine using conventional technique: computed tomography postoperative assessment in 102 consecutive patients. J Neurosurg Spine. 2010;12:306-13.

18. Kim MC, Chung HT, Cho JL, Kim DJ, Chung NS. Factors affecting the accurate placement of percutaneous pedicle screws during minimally invasive transforaminal lumbar interbody fusion. Eur Spine J. 2011;20:1635-43.

19. Nojiri K, Matsumoto M, Chiba K, Toyama Y. Morphometric analysis of the thoracic and lumbar spine in Japanese on the use of pedicle screws. Surg Radiol Anat. 2005;27:123-8.

20. Nojiri K, Matsumoto M, Chiba K, Toyama Y, Momoshima S. Comparative assessment of pedicle morphology of the lumbar spine in various degenerative diseases. Surg Radiol Anat. 2005;27:317-21.

21. Wiesner L, Kothe R, Schulitz KP, Ruther W. Clinical evaluation and computed tomography scan analysis of screw tracts after percutaneous insertion of pedicle screws in the lumbar spine. Spine (Phila Pa 1976). 2000;25:615-21.

22. Vaidya R, Carp J, Bartol S, Ouellette N, Lee S, Sethi A. Lumbar spine fusion in obese and morbidly obese patients. Spine (Phila Pa 1976). 2009;34:495-500.

23. Kim YJ, Lenke LG, Cheh G, Riew KD. Evaluation of pedicle screw placement in the deformed spine using intraoperative plain radiographs: a comparison with computerized tomography. Spine (Phila Pa 1976). 2005;30:2084-8.

24. Cui G, Wang Y, Kao TH, Zhang Y, Liu Z, Liu B, et al. Application of intraoperative computed tomography with or without navigation system in surgical correction of spinal deformity: a preliminary result of 59 consecutive human cases. Spine (Phila Pa 1976). 2012;37:891-900.

25. Wu ZX, Huang LY, Sang HX, Ma ZS, Wan SY, Cui G, et al. Accuracy and safety assessment of pedicle screw placement using the rapid prototyping technique in severe congenital scoliosis. J Spinal Disord Tech. 2011:24:444-50.

26. Farber GL, Place HM, Mazur RA, Jones DE, Damiano TR. Accuracy of pedicle screw placement in lumbar fusions by plain radiographs and computed tomography. Spine (Phila Pa 1976). 1995:20:1494-9.

\section{Submit your next manuscript to BioMed Central and take full advantage of:}

- Convenient online submission

- Thorough peer review

- No space constraints or color figure charges

- Immediate publication on acceptance

- Inclusion in PubMed, CAS, Scopus and Google Scholar

- Research which is freely available for redistribution 